patients with 3 or more stations sampled varied from $0 \%$ to $26.6 \%$. Furthermore, there was variability in the rate of inadequate sampling (2.4-7.6\%), sensitivity (69.2-90.5\%), negative predictive value $(50-87.5 \%)$ and rate of surgical sampling of negative nodes (6.3-35.0\%) across the EBUS centres.

Discussion There is variability in practice in all parameters of EBUS practice examined in our Network, in the absence of agreed standards and protocols for mediastinal staging. Such protocols and standards have now been agreed and implemented across our Network and the EBUS sub-group is committed to ongoing data collection and publication to drive quality outcomes in this pivotal lung cancer service.

\section{S72 CLINICAL PREDICTION MODELS FOR MALIGNANCY IN SOLITARY PULMONARY NODULES - A VALIDATION STUDY IN A UK POPULATION}

${ }^{1}$ Ali Al-Ameri, ${ }^{2}$ Puneet Malhotra, ${ }^{3}$ Helene Thygesen, ${ }^{1}$ Sri Vaidyanathan, ${ }^{4}$ Paul Plant, 'Shishir Karthik, 'Andrew Scarsbrook, 'Matthew Callister. 'St James's University Hospital, Leeds, UK; ${ }^{2}$ Whiston Hospital, Prescot, UK; ${ }^{3}$ Leeds Institute of Cancer and Pathology, Leeds, UK; ${ }^{4}$ North Cumbria's Hospital, UK

\subsection{6/thoraxjnl-2014-206260.78}

Background Management of solitary pulmonary nodules (SPNs) depends critically on the pre-test probability of malignancy. Several quantitative prediction models have been developed using clinical and radiological criteria. Three models include CT criteria (Mayo, Veterans Association, Brock University) with a fourth model (Herder) incorporating FDG avidity on CT-PET scan in addition. These models have not been validated in a UK population, and the current study aimed to compare their performance in a population of patients recruited from a UK teaching hospital.

Methods Patients with SPNs $(4-30 \mathrm{~mm})$ were retrospectively identified from the lung cancer MDT and a nodule follow-up clinic $(\mathrm{n}=246)$. All patients had a final diagnosis confirmed by histology or radiological stability on a 2-year follow up. For each patient, the probability of the pulmonary nodule being malignant was calculated using the four models described. The models were used both in a restricted cohort of patients based

Malignancy prediction models comparison

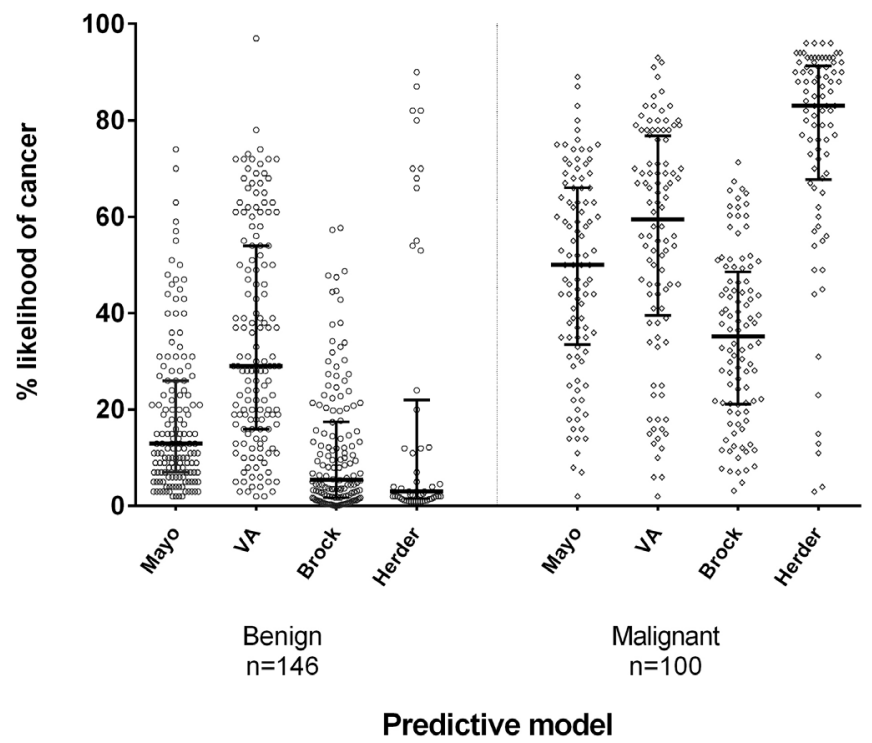

Abstract S72 Figure 1 Malignancy prediction models comparison on their respective exclusion criteria, and in the total cohort of patients. The accuracy of each model was assessed by calculating the area under the receiver operating characteristic (ROC) curve. Results The median age of the patient population was 69 years (range 32-94) and 50\% were male. The prevalence of malignancy was $40.6 \%$ (33.3\% primary lung cancer, $7.3 \%$ metastatic disease). Figure 1 shows the distribution of the probabilities of malignancy according to the four different models.

The areas under the ROC curves for the cohorts restricted by respective exclusion criteria were (AUC, 95\% CI): Mayo 0.892 (0.847-0.937); VA 0.736 (0.672-0.801); Brock 0.901 (0.855$0.947)$ and Herder $0.924(0.875-0.974)$. For the total cohort, the AUC values were Mayo 0.873, VA 0.736, Brock 0.867 and Herder 0.916.

There was no statistical difference between the Mayo and Brock models, but both were significantly better than VA (AUC difference of 0.14 and 0.13 respectively, $\mathrm{p} \leq 0.0001$ for both). The Herder model performed significantly better than both Mayo and Brock models (AUC difference of 0.10 and 0.14 respectively, $\mathrm{p} \leq 0.01$ ).

Conclusion Both the Mayo and the Brock models perform well in a UK population, but accuracy is improved by incorporating CT-PET findings using the Herder prediction model.

\section{S73 INFRARED SPECTROSCOPY FOR THE DETECTION OF EXTENDED FIELD CARCINOGENESIS: A NEW PARADIGM FOR LUNG CANCER SCREENING?}

JM Brown, L Foreman, K Oliver, R Thakrar, A Maréchal, P Rich, SM Janes. University College London, London, UK

\subsection{6/thoraxjnl-2014-206260.79}

Background Computerised Tomography (CT) has been shown to be the only lung cancer screening modality to be effective in reducing lung cancer specific mortality. ${ }^{1}$ A minimally invasive technique to stratify those at greatest risk within the population of adult smokers may help to target CT screening more effectively.

Rationale Tobacco smoke exposure causes a field of injury to the airways (including nose and mouth) that if detected may inform an individual's risk of lung cancer. ${ }^{2}$ Infrared spectroscopy (IR) is a technique that can detect subtle biochemical alterations in macroscopically normal cells.

Methods Buccal cells were exfoliated from 76 patients including 38 smokers without and 38 with lung cancer (matched for age, gender and pack years). The cells were fixed onto IR windows and spectra recorded using synchrotron radiation (Diamond facility, Oxford). Data was acquired using x36 objective and $15 \times 15 \mu \mathrm{m}$ aperture in transmission mode; 256 interferograms at $4 \mathrm{~cm}^{-1}$ resolution were recorded for 50 cells per sample. All samples analysed were confirmed to be cytologically normal. Outlying data was removed using principal component analysis and a prediction model built using partial least squares discriminant analysis.

Results Smokers with lung cancer could be differentiated from matched smokers without lung cancer with a diagnostic accuracy of $80 \%$. The spectral region showing greatest difference between groups was in the $1200-900 \mathrm{~cm}^{-1}$ region; comparison to reference spectra shows that this is likely to represent a metabolic change caused by an increased abundance of glycogen or its derivatives.

Conclusions We have shown for the first time that IR spectroscopy of macroscopically normal upper respiratory tract cells may have a role to play in the early detection of lung cancer. Future work will validate these findings and aim to develop this 